\begin{tabular}{|c|l|}
\hline Title & DJ-1 binds to mitochondrial complex I and maintains its activity \\
\hline Author(s) & $\begin{array}{l}\text { Hayashi, Takuy a; Ishimori, Chikako; Takahashi-Niki, Kazuko; Taira, Takahiro; Kim, Y un-chul; Maita, Hiroshi; Maita, } \\
\text { Chinatsu; A riga, Hiroy oshi; Iguchi-A riga, Sanae M. M. }\end{array}$ \\
\hline Citation & $\begin{array}{l}\text { Biochemical and Biophysical Research Communications, 390(3), 667-672 } \\
\text { https://doi.org/10.1016/.bbrc.2009.10.025 }\end{array}$ \\
\hline Issue Date & 2009-12-18 \\
\hline Doc URL & http://hdl.handle.net/2115/42484 \\
\hline Type & article (author version) \\
\hline File Information & BBRC390-3_667-672.pdf \\
\hline
\end{tabular}

Instructions for use 


\section{DJ-1 binds to mitochondrial complex I and maintains its activity}

Takuya Hayashi $^{\mathrm{a}}$, Chikako Ishimori ${ }^{\mathrm{a}}, \underline{\text { Kazuko Takahashi-Niki }^{\mathrm{c}}, \text { Takahiro Taira }^{\mathrm{b}} \text {, Yun-chul }}$

$\underline{\text { Kim }}^{\mathrm{c}}$, Hiroshi Maita ${ }^{\mathrm{c}}$, Chinatsu Maita ${ }^{\mathrm{a}}$, Hiroyoshi Ariga ${ }^{\mathrm{c}}$,, Sanae M.M. Iguchi-Ariga ${ }^{\mathrm{a}}$

${ }^{\mathrm{a}}$ Graduate School of Agriculture, Hokkaido University, Sapporo 060-8589, Japan

${ }^{\mathrm{b}}$ Interdisciplinary Graduate School of Medicine and Engineering, Yamanashi University,

Chuoh, 409-3898, Japan

${ }^{\mathrm{c}}$ Graduate School of Pharmaceutical Sciences, Hokkaido University, Sapporo 060-0812, Japan

*Correspondence author. Address: Graduate School of Pharmaceutical Sciences, Hokkaido University, Sapporo 060-0812, Japan. Fax: +81 117063745.

E-mail address: hiro@pharm.hokudai.ac.jp (H. Ariga) 


\begin{abstract}
Parkinson's disease (PD) is caused by neuronal cell death, and oxidative stress and mitochondrial dysfunction are thought to be responsible for onset of PD. DJ-1, a causative gene product of a familial form of Parkinson's disease, PARK7, plays roles in transcriptional regulation and anti-oxidative stress. The possible mitochondrial function of DJ-1 has been proposed, but its exact function remains unclear. In this study, we found that DJ-1 directly bound to NDUFA4 and ND1, nuclear and mitochondrial DNA-encoding subunits of mitochondrial complex I, respectively, and was co-localized with complex I and that complex I activity was reduced in DJ-1-knockdown NIH3T3 and HEK293cells. These findings suggest that DJ-1 is an integral mitochondrial protein and that DJ-1 plays a role in maintenance of mitochondrial complex I activity.
\end{abstract}

Key words: DJ-1; mitochondria; Parkinson's disease; complex I 


\section{Introduction}

Parkinson's disease (PD) is caused by dopaminergic cell death, and genetic and environmental factors are thought to affect the onset of PD. These factors lead to oxidative stress, mitochondrial dysfunction and impairment of the protein degradation system, resulting in cell death. DJ-1 was first identified by our group as a novel oncogene product [1] and was later found to be a causative gene product of a familial form of PD, PARK7 [2]. DJ-1 plays roles in transcriptional regulation [3-8] and anti-oxidative stress reaction [9-12], and loss of its function is thought to affect the onset of PD. DJ-1 has three cysteines at amino acid numbers 46, 53 and 106 (C46, C53 and C106, respectively). Although oxidation of C106 is necessary for DJ-1 to exert its activity [11-13], further oxidation of C106 is thought to render DJ-1 inactive [14], and such oxidized DJ-1 has been observed in patients with the sporadic form of PD and Alzheimer disease $[15,16]$.

Reduced activity level and reduced amount of mitochondrial complex I have been reported in PD patients [17-21] and in animal models [22, 23]. These findings indicate that the function of mitochondrial complex I is related to the onset of PD. DJ-1 is located both in the cytoplasm and nucleus [1] and has recently been shown to be located in mitochondria [11, 24-26]. It has also been reported that some parts of DJ-1 were translocated into mitochondria after cells had been subjected to oxidative stress to protect cells from oxidative stress-induced cell death [27-30]. The exact localization of DJ-1 in mitochondria, inner membrane, outer membrane or intermembrane space, is, however, still not clear, and the characteristics and function of mitochondria-resident DJ-1 are still not known.

In this study, we analyzed the effect of DJ-1 on mitochondrial function. The results showed that DJ-1 was bound to complex I and that knockdown of DJ-1 in cells decreased complex I 
activity.

\section{Materials and methods}

\section{Cell culture}

Human SH-SY5Y, human 293, mouse NIH3T3 and D2 cells were cultured in Dulbecco's modified Eagle's medium (DMEM) with 10\% calf serum. D2 cells are NIH3T3 cells in which the expression of DJ-1 has been knocked down by shRNA targeting the mouse $D J-1$ gene as described previously [17]. HL-60 cells were cultured in RPMI1640 medium with $10 \%$ fetal calf serum.

\section{$\underline{\text { Establishment of DJ-1-knockdown cell lines }}$}

The nucleotide sequence of the upper strand of the oligonucleotide used for construction of an siRNA vector targeting the human $D J-1$ gene is 5 '-

\section{GGATCCCGTTTATCTGAGTCTGCTGCTTTCAAGAGAAGCAGCAGACTCAGATAAAT}

TTTTTCCAAAAGCTT-3'. After annealing oligonucleotides corresponding to the upper and lower strands of DNA, they were inserted into BamHI-HindIII sites of pRNA-H1-tet/Hygro (GenScript Corp., Piscataway, NJ, USA). These plasmids were transfected into human T-REx-293 cells (Invitrogen, Carlsbad, CA, USA) by the calcium phosphate precipitation method, and the cells were cultured in the medium in the presence of $400 \mu \mathrm{g} / \mathrm{ml}$ hygromycin for 14 days. Cells that were resistant to the drug were then selected. These cells were named Tet-DJ-1-knockdown cells. To establish cell lines harboring wild-type DJ-1 or L166P DJ-1, plasmid pcDNA3 containing Flag-tagged wild-type or L166P DJ-1was transfected into D2 $\underline{\text { cells }}$, and the cells were cultured in a medium in the presence of $\underline{400 \mu \mathrm{g} / \mathrm{ml} \text { hygromycin}}$, 
respectively, for 14 days. Cells resistant to the drug were then selected.

Cloning of DJ-1-binding proteins by a two-hybrid system

The two-hybrid screening using human brain MATCHMAKER cDNA (Clontech, Mountain View, USA) was described previously [3].

\section{Activity of mitochondrial complex I}

NIH3T3, D2 and Tet-DJ-1-kockdown cells were used. To induce DJ-1-knockdown in

Tet-DJ-1-knockdown cells, cells were cultured in the medium with $1 \mu \mathrm{g} / \mathrm{ml}$ doxycycline for 72 hrs. Cells were suspended in a buffer containing $0.25 \mathrm{M}$ sucrose and their extracts were prepared by homogenization using a Potter type of homogenizer. The mitochondria were pelleted after centrifugation of extracts at $12,000 \mathrm{x} g$ for $15 \mathrm{~min}$, suspended in the buffer containing $0.25 \mathrm{M}$ sucrose and used as the mitochondria-rich fraction. Activity of complex I was measured by reacting the mitochondria in a buffer containing $6.65 \mathrm{mM} \mathrm{NaH}_{2} \mathrm{PO}_{4}, 28.35$ $\mathrm{mM} \mathrm{Na} 2 \mathrm{HPO}_{4}, 5 \mathrm{mM} \mathrm{MgCl} 2,5 \mathrm{mM}$ EDTA, $1 \mathrm{mg} / \mathrm{ml} \mathrm{BSA}, 2 \mathrm{ng} / \mathrm{ml}$ antimycine, $50 \mu \mathrm{M}$ ubiquinone 1 and $2.65 \mathrm{mM} \mathrm{NaN}_{3}$ for $2 \mathrm{~min}$ at $37^{\circ} \mathrm{C}$. After the reaction, $5 \mathrm{mM} \mathrm{NADA}$ was added to the mixture and its absorbance at $340 \mathrm{~nm}$ was measured.

\section{Western blotting}

Preparations of proteins and conditions for Western blotting were described previously $[3,9]$. After proteins were reacted with primary antibodies, proteins on the membrane were reacted with an IRDye800 (Rockland, Philadelphia, PA, USA) or Alexa680-conjugated secondary antibody (Molecular Probe, Eugene, OR, USA) and visualized by using an infrared imaging 
system (Odyssey, LI-COR, Lincoln, NE). Primary antibodies used were anti-NDUFA9 (1:1,000, MitoScience, Eugene, OR, USA), anti-NDUFS3 (1:1,000, Molecular Probe), anti-ND6 (1:1,000, MitoScience), anti-ND1 (1:1000, Santa Cruz), anti-ATP5A1(1:1,000, MitoScience), anti-actin (1:4,000, Chemicon, Temecula, CA, USA), and anti-DJ-1 (1:4,000) antibodies. The anti-DJ-1 antibody was prepared by us as described previously [1].

\section{Immunofluorescence}

Cells were cultured in DMEM containing $100 \mathrm{nM}$ MitoTracker Red CMXRos (Molecular Probe) for $45 \mathrm{~min}$. The medium was replaced with DMEM alone and cultured for $3 \mathrm{hrs}$. Cells were then fixed with $4 \%$ paraformaldehyde for $10 \mathrm{~min}$ followed by incubation with $0.1 \%$ Triton X-100 in PBS for 10 min and stained with a rat anti-DJ-1 antibody (1:10). After reaction of cells with an FITC-conjugated anti-rat antibody, nuclei were stained with DAPI (1:1000), and cell images were visualized by using a fluorescence microscope (BZ-8000, Keyence). To see mitochondria-resident DJ-1, NIH3T3 cells were fixed with 4\% paraformaldehyde for 20 min, washed with PBS and then heated at $95^{\circ}$ for $15 \mathrm{~min}$ in a buffer containing $100 \mathrm{mM}$ Tris- $\mathrm{HCl}(\mathrm{pH} 9.5)$ and 5\% urea. Cells were then incubated with $0.1 \%$ Triton X-100 in PBS for 10 min and stained with rabbit anti-DJ-1 (1:400) and mouse anti-NDUFS3 (1:100, Molecular Probe) antibodies. After reaction of cells with FITC-conjugated anti-rabbit and rhodamine-conjugated anti-mouse antibodies, nuclei were stained with DAPI (1:1000), and cell images were visualized by using a confocal laser microscope (Zeiss LSM510).

In vitro binding assay (pull-down assay) and in vivo binding assay 
Conditions for pull-down assays using ${ }^{35}$ S-labeled NDUFA4 and GST-DJ-1 and in vivo binding assays were described previously [3, 4].

\section{Statistical evaluation}

All data are shown as mean \pm standard error of the mean (SEM). The significance of differences was determined by one-way or repeated measures analysis of variance (ANOVA). Further statistical analysis was performed using the Mann-Whitney U-test.

\section{Results}

\section{Identification of subunits of mitochondrial complex I as DJ-1-binding proteins}

During the course of isolation of DJ-1-binding proteins by a two-hybrid screening using a human brain cDNA library, we identified NDUFA4 and ND1/MT-ND1, which are nuclear and mitochondrial DNA-encoding subunits, respectively, of the mitochondrial membrane respiratory chain NADH dehydrogenase (complex I). To confirm association of DJ-1 with NDUFA4 and examine the effect of oxidative stress on binding in cells, human 293T cells $\underline{\text { were transfected with an expression vector for Flag-tagged NDUFA4 and exposed to } 200 \mu \mathrm{M}}$ $\underline{\mathrm{H}}_{2} \underline{\mathrm{O}}_{2} \underline{\text { for } 2 \mathrm{hrs} \text { at } 48 \mathrm{hrs} \text { after transfection. Proteins were then immunoprecipitated with an }}$ anti-Flag antibody or non-specific IgG, and precipitates were analyzed by Western blotting with anti-Flag and anti-DJ-1 antibodies (Fig. 1A). To separate reduced and oxidized forms of DJ-1, proteins were separated on 10-20\% gradient of polyacrylamide gels, in which an oxidized form of DJ-1 run slowly compared to the reduced form of DJ-1. The results clearly $\underline{\text { showed that Flag-NDUFA4 was associated with both reduced and oxidized forms of }}$ endogenous DJ-1 and that its association was enhanced by oxidative stress. To examine direct 
interaction of DJ-1 with NDUFA4, pull-down assays using purified GST-DJ-1, GST and

${ }^{35}$ S-labeled NDUFA4, which had been synthesized in vitro, were carried out. As shown in Fig. $1 \mathrm{~B},{ }^{35} \mathrm{~S}$-labeled NDUFA4 was found to bind to GST-DJ-1 but not GST, indicating direct binding of DJ-1 with NDUFA4. To examine the association of DJ-1 with ND1 and the effect of oxidative stress on its association, human 293 cells or human neuroblastoma cell line $\underline{\text { SH-SY5Y were exposed to } 200 \mu \mathrm{M} \mathrm{H}_{2} \underline{\mathrm{O}}_{2} \text { for } 2 \mathrm{hrs} \text {. Proteins extracted from cells were then }}$ immunoprecipitated with an anti-DJ-1 antibody or non-specific IgG, and precipitates were analyzed by Western blotting with anti-DJ-1 and anti-ND1 antibodies (Fig. 1C and 1D). The results clearly showed the association of DJ-1 with endogenous ND1 and enhancement of its $\underline{\text { association by oxidative stress. }}$ To assess endogenous association of DJ-1 with complex I, proteins from human HL60 cells were immunoprecipitated with an anti-DJ-1 antibody and precipitates were analyzed by Western blotting with an anti-NDUFS3 antibody (Fig. 1E). NDUFS3 is a core subunit of complex I and was found to be associated with DJ-1. Since ND1 is mitochondrial DNA-encoding protein and synthesized within mitochondria using mitochondrial ribosome, these results showed that DJ-1 was associated with complex I in mitochondria.

Localization of DJ-1 in cells was then examined by immunofluorescence techniques. First, SH-SY5Y cells were stained both with an anti-DJ-1 antibody and with Mitotracker Red, which indicates the location of mitochondria. Nuclei were stained with DAPI (Fig. 2A). As described previously $[1, \underline{26,28}]$, DJ-1 was located both in the nucleus and cytoplasm and some portions of DJ-1 were colocalized with mitochondria under a non-stress condition (See merged figure in which green (DJ-1) and red (mitochondria) were turned yellow.). To clearly see mitochondria-resident proteins, NIH3T3 cells on cover glasses were heated to inactivate 
antigenecity of cytoplasmic proteins and then stained with anti-DJ-1 and anti-NDUFS3

antibodies. As shown in Fig. 2B, DJ-1 and NDUFS3 were completely colocalized, indicating localization of DJ-1 on/in complex I.

\section{Reduction of complex I activity in DJ-1-knockdown cells}

Since DJ-1 was associated with complex I, it is possible that DJ-1 affects complex I activity. Expression levels of DJ-1 and subunits of mitochondrial complexes in NIH3T3 cells and their knockdown cells D2 were first examined by Western blotting. About $80 \%$ reduced expression of DJ-1 was observed in D2 cells compared to the expression of DJ-1 in NIH3T3 cells (Fig. 3B). Difference of expression levels of three subunits of complex I, NDUFA9, NDUFS3 and ND6, and a subunit of complex V, ATP5A1, between D2 and NIH3T3 cells were much smaller than that of DJ-1. Activities of complex I in these cells were then measured, and it was found that the activity in D2 cells was only about 30\% of that in NIH3T3 cells (Fig. 3A).

To compare complex I activities under the same conditions, human 293 cells expressing the tetracycline-responsible repressor were transfected with shRNA targeting DJ-1 that was linked to the tetracycline-responsible promoter, and DJ-1-knockdown cells were established by addition of doxycycline. These cells were named Tet-DJ-1-knockdown cells and the expression level of DJ-1 in these cells was examined. After addition of $1 \mu \mathrm{g} / \mathrm{ml}$ of doxycycline, the expression level of DJ-1 decreased in a time-dependent manner and about $70 \%$ reduction of DJ-1 expression was observed at 72 hrs after addition of doxycycline (Fig. 3C, left panel) and its quantified data (Fig. 3C, right panel)). While complex I activity in 293 cells harboring a vector without shRNA (vector cells) was not changed, complex I activity in Tet-DJ-1-knockdown cells was found to be about $50 \%$ of that in cells without addition of 
doxycycline (Fig. 3D). Expression levels of ATG5A1 and actin, but not DJ-1, in both types of cells were not changed under this condition (Fig. 3E). The results obtained in D2 and Tet-DJ-1-knockdown cells suggest that reduction of DJ-1 expression attenuates complex I activity without affecting integrity of mitochondrial complexes.

To examine the association of L166P DJ-1, which is mutant DJ-1 found in PD patient [2], with NDUFA4, HA-tagged wild-type or L166P DJ-1 and Flag-tagged NDUFA4 were co-transfected into $293 \mathrm{~T}$ cells. Forty-eight hrs after transfection, proteins extracted from cells were immunoprecipitated with an anti-Flag antibody and precipitates were analyzed by Western blotting with an anti-HA antibody (Fig. 4A). The results showed that L166P DJ-1 bound to NDUFA4 with intensity similar to that of wild-type DJ-1.To confirm a role of DJ-1 in maintenance of complex 1 activity, D2 cells expressing wild-type DJ-1 or L166P DJ-1 were established and their complex 1 activity was measured. The results showed that complex I activity was partially, but not completely, restored with wild-type DJ-1 but not with L166P DJ-1 (Fig. 4B), suggesting that DJ-1 is necessary for maintenance of complex 1 activity but that the other factor(s) may be required to exert full activity of complex 1

\section{Discussion}

In the present study, we showed that DJ-1 was colocalized with mitochondrial complex I even under a non-stress condition and that DJ-1 directly bound to NDUFA4, a subunit of complex I that is localized in a mitochondrial inner membrane. Binding of DJ-1 to subunits of complex I $\underline{\text { was enhanced by oxidative stress. These findings suggest that native DJ-1 prevents }}$

$\underline{\text { Parkinson's disease via maintaining mitochondrial complex I activity through NDFU4 and }}$

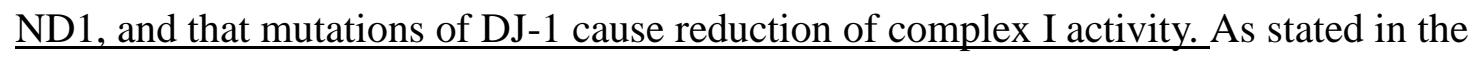


Introduction section, the localization of DJ-1 in mitochondria is still not clear. While Zhang et al. reported that DJ-1 is located in the mitochondrial matrix and intermembrane space [24], Canet-Aviles et al. reported that DJ-1 is located in the outer membrane but not inside of mitochondrial outer membrane [11]. Although the reasons for this discrepancy are not clear, different cells and methods used to identify the localization of DJ-1 might have given rise to the discrepancy. NDUFA4 is an accessory subunit of mitochondrial complex I, encoded by the nuclear gene and localized in the peripheral membrane at the matrix side of the mitochondrial inner membrane. ND1 is the subunit of mitochondrially encoded NADH dehydrogenase 1 and synthesized within mitochondria using mitochondrial translation machinery. Since binding of DJ-1 to ND1 and NDUFA4 and complete colocalization of DJ-1 with mitochondrial complex I were, however, shown in this study, at least some portion of DJ-1 is thought to be localized in the mitochondrial inner membrane.

Complex I activity was then found to decrease in two types of DJ-1-knockdown cells, mouse NIH3T3 and human 293 cells, in which mitochondrial integrity was maintained. When wild-type DJ-1 was re-introduced into D2 cells, which are DJ-1-knockdown NIH3T3 cells, and complex I activity of these cells was measured, it was found that complex I activity was partially, but not completely, restored compared with that in parental NIH3T3 cells. These results suggest that DJ-1 is necessary for mitochondrial complex I to exert its enzyme activity but that DJ-1 alone is not sufficient to maintain activity of complex I. Knockdown of DJ-1 expression may induce a change in structure, but not amounts of components, of complex I, resulting in reduced complex I activity. Alternatively, it is possible that DJ-1 plays a role in the assembly of complex I. Reduction of DJ-1 expression therefore influences complex I activity. Furthermore, when isolated mitochondria prepared from normal or DJ-1-knockdown 
cells were mixed with recombinant wild-type DJ-1 and then their complex I activity was measured, no stimulation of complex I activity was observed (data not shown). These results suggest possibilities described above or suggest that DJ-1 alone is not translocated into complex I. Since DJ-1 possesses no mitochondrial import signal, it is thought that DJ-1 is translocated into complex I with association of another protein(s) that possesses mitochondrial import signals.

Taken together, the present findings provide insights into molecular mechanisms not only of the onset of Parkinson's disease but also of the regulation of mitochondrial complex I.

\section{Acknowledgments}

We thank Kiyomi Takaya for her technical assistance. This work was supported by grants-in-aid from the Ministry of Education, Science, Culture and Sports and by the Program for Promotion of Fundamental Studies in Health Sciences of the National Institute of Biomedical Innovation (NIBIO) in Japan. 


\section{References}

[1] D. Nagakubo, T. Taira, H. Kitaura, M. Ikeda, K. Tamai, S.M.M. Iguchi-Ariga, H. Ariga, DJ-1, a novel oncogene which transforms mouse NIH3T3 cells in cooperation with ras, Biochem. Biophys. Res. Commun. 231 (1997) 509-513.

[2] V. Bonifati, P. Rizzu, M.J. van Baren et al, Mutations in the DJ-1 gene associated with autosomal recessive early-onset parkinsonism, Science 299 (2003) 256-259.

[3] K. Takahashi, T. Taira, T. Niki, C. Seino, S.M.M. Iguchi-Ariga, H. Ariga, DJ-1 positively regulates the androgen receptor by impairing the binding of PIASx alpha to the receptor, J. Biol. Chem. 276 (2001) 37556-37563.

[4] T. Niki, K. Takahashi-Niki, T. Taira, S.M.M. Iguchi-Ariga, H. Ariga H, DJ-1-binding protein, negatively regulates the androgen receptor by recruiting histone deacetylase complex, and DJ-1 antagonizes this inhibition by abrogation of this complex, Mol. Cancer Res. 1(2003) 247-261.

[5] Y. Shinbo, T. Taira, T. Niki, S.M.M. Iguchi-Ariga, H. Ariga, DJ-1 restores p53 transcription activity inhibited by Topors/p53BP3, Int. J. Oncol. 26 (2005) 641-648.

[6] N. Zhong, C.Y. Kim, P. Rizzu, C. Geula, D.R. Porter, E,N, Pothos, F. Squitieri, P. Heutink, J. Xu, DJ-1 transcriptionally up-regulates the human tyrosine hydroxylase by inhibiting the sumoylation of pyrimidine tract-binding protein-associated splicing factor, $\mathrm{J}$. Biol. Chem. 281 (2006) 20940-20948.

[7] C.M. Clements, R.S. McNally, B.J. Conti, T.W. Mak, J.P Ting, DJ-1, a cancer- and Parkinson's disease-associated protein, stabilizes the antioxidant transcriptional master regulator Nrf2, Proc. Natl. Acad. Sci. USA. 103 (2006) 15091-15096.

[8] J.E. Tillman, J. Yuan, G. Gu, L. Fazli, R. Ghosh, A.S. Flynt, M. Gleave, P.S. Rennie, S. 
Kasper, DJ-1 binds androgen receptor directly and mediates its activity in hormonally treated prostate cancer cells, Cancer Res. 67 (2007) 4630-4637.

[9] T. Taira, Y. Saito, T. Niki, S.M.M. Iguchi-Ariga, H. Ariga, DJ-1has a role in anti-oxidative stress to prevent cell death, EMBO Rep. 5 (2004) 213-218.

[10] T. Yokota, K. Sugawara, K. Ito, R. Takahashi, H. Ariga, H. Mizusawa, Down regulation of DJ-1 enhances cell death by oxidative stress, ER stress, and proteasome inhibition, Biochem. Biophys. Res. Commun. 312 (2003) 1342-1348.

[11] R.M. Canet-Aviles, M.A. Wilson, D.W. Miller, R. Ahmad, C. McLendon, S. Bandyopadhyay, M.J. Baptista, D. Ringe, G.A. Petsko, M.R. Cookson, The Parkinson's disease protein DJ-1 is neuroprotective due to cysteine-sulfinic acid-driven mitochondrial localization, Proc. Natl. Acad. Sci. USA. 101 (2004) 9103-9108.

[12] C. Martinat, S. Shendelman, A. Jonason, T. Leete, M.F. Beal, L. Yang, T. Floss, A. Abeliovich A, Sensitivity to oxidative stress in DJ-1-deficient dopamine neurons: an ES-derived cell model of primary parkinsonism, PLoS Biol. 2 (2004) e327.

[13] K. Takahashi-Niki, T. Niki, T. Taira, S.M.M. Iguchi-Ariga, H. Ariga, Reduced anti-oxidative stress activities of DJ-1 mutants found in Parkinson's disease patients, Biochem. Biophys. Res. Commun. 320 (2004) 389-397.

[14] W. Zhou, M. Zhu, M.A. Wilson, G.A. Petsko, A.L. Fink, The oxidation state of DJ-1 regulates its chaperone activity toward alpha-synuclein, J. Mol. Biol. 356 (2006) 1036-1048.

[15] R. Bandopadhyay, A.E. Kingsbury, M.R. Cookson, et al, The expression of DJ-1 (PARK7) in normal human CNS and idiopathic Parkinson's disease, Brain 127 (2004) 420-430. 
[16] J, Choi, M.C. Sullards, J.A. Olzmann, H.D. Rees, S.T. Weintrau', D.E. Bostwick, M. Gearing, A.I. Levey, L.-S. Chin, L. Li, Oxidative damage of DJ-1 is linked to sporadic Parkinson and Alzheimer diseases, J. Biol. Chem. 281 (2006) 10816-10824.

[17] Y. Mizuno, S.Ohta, M. Tanaka, S. Takamiya, K. Suzuki, T. Sato, H. Oya, T. Ozawa, Y. Kagawa, Deficiencies in complex I subunits of the respiratory chain in Parkinson's disease, Biochem. Biophys. Res. Commun. 163 (1989) 1450-1455.

[18] W.D. Parker Jr., S.J. Boyson, J.K. Parks, Abnormalities of the electron transport chain in idiopathic Parkinson's disease, Ann. Neurol. 26 (1989) 719-723.

[19] A.H. Schapira, J.M. Cooper, D. Dexter, J.B. Clark, P. Jenner, C.D. Marsden, Mitochondrial complex I deficiency in Parkinson's disease, J. Neurochem. 54 (1990) 823-827.

[20] M. Orth, A.H. Schapira, Mitochondrial involvement in Parkinson's disease, Neurochem. Int. 40 (2002) 533-541.

[21] Y. Mizuno, N. Hattori, H. Matsumine, Neurochemical and neurogenetic correlates of Parkinson's disease, J. Neurochem. 71 (1998) 893-902.

[22] W.J. Nicklas, I. Vyas, R.E. Heikkila, Inhibition of NADH-linked oxidation in brain mitochondria by 1-methyl-4-phenyl-pyridine, a metabolite of the neurotoxin, 1-methyl-4-phenyl-1,2,5,6-tetrahydropyridine, Life Sci. 36 (1985) 2503-2508.

[23] L. Flinn, H. Mortiboys, K. Volkmann, R.W. Köster, P.W. Ingham, O. Bandmann, Complex I deficiency and dopaminergic neuronal cell loss in parkin-deficient zebrafish (Danio rerio), Brain 132 (2009) 1613-1623.

[24] S. Shendelman, A. Jonason, C. Martinat, T. Leete, A. Abeliovich, DJ-1 is a redox-dependent molecular chaperone that inhibits alpha-synuclein aggregate formation, 
PLoS Biol. 2 (2004) e362.

[25] L. Zhang, M. Shimoji, B. Thomas, et al, Mitochondrial localization of the Parkinson's disease related protein DJ-1: implications for pathogenesis, Hum. Mol. Genet. 14 (2005) 2063-2073.

[26] Y. Shinbo, T. Niki, T. Taira, H. Ooe, K. Takahashi-Niki, C. Maita, C. Seino, S.M.M. Iguchi-Ariga, H. Ariga, Proper SUMO-1 conjugation is essential to DJ-1 to exert its full activities, Cell Death Diff. 13 (2006) 96-108.

[27] H. Ooe, T. Taira, S.M.M. Iguchi-Ariga, H. Ariga, Induction of reactive oxygen species by bisphenol A and abrogation of bisphenol A-induced cell injury by DJ-1, Toxicol. Sci. 88 (2005) 114-126.

[28] H.M. Li, T. Niki, T. Taira, S.M.M. Iguchi-Ariga, H. Ariga, Association of DJ-1 with chaperones and enhanced association and colocalization with mitochondrial Hsp70 by oxidative stress, Free Radic. Res. 39 (2005) 1091-1099.

[29] N. Lev, D. Ickowicz, E. Melamed, D. Offen, Oxidative insults induce DJ-1 upregulation and redistribution: implications for neuroprotection, NeuroToxicology 29 (2008) 397-405.

[30] E. Junn, W.E. Jang, X. Zhao, B. Jeong, M.M. Mouradian, Mitochondrial localization of DJ-1 leads to enhanced neuroprotection, J. Neurosci. Res. 87 (2009) 123-129. 


\section{Legends of figures}

\section{Fig. 1. Association of DJ-1 with mitochondrial complex I.}

A. 293T cells were transfected with an expression vector for Flag-NDUFA4 and exposed to $\underline{200 \mu \mathrm{M} \mathrm{H}_{2}} \underline{\mathrm{O}}_{2}$ for $2 \mathrm{hrs}$ at $48 \mathrm{hrs}$ after transfection. Proteins in extracts were immunoprecipitated with an anti-Flag antibody or IgG and precipitates were analyzed by Western blotting with anti-DJ-1 and anti-Flag antibodies as described in Materials and methods.

B. Upper panel: Pull-down assays were carried out using purified GST, GST-DJ-1 and

${ }^{35}$ S-labeled NDUFA4 that had been synthesized in vitro. After the reaction, proteins were analyzed by fluorography as described in Materials and methods. Lower panel: Gels used for separation of pulled-down proteins were stained with CBB.

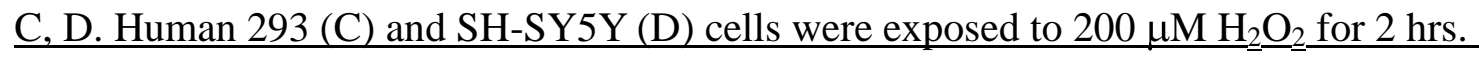
Proteins were then extracted from cells and immunoprecipitated with an anti-DJ-1 antibody or $\underline{\text { IgG and precipitates were analyzed by Western blotting with anti-DJ-1 and anti-ND1 }}$ antibodies.

E. Proteins were extracted from HL60 cells. Proteins in extracts were immunoprecipitated with an anti-DJ-1 antibody or IgG and precipitates were analyzed by Western blotting with anti-DJ-1 and anti-NDUFS3 antibodies. IgG-Hc indicates heavy chain of IgG.

\section{Fig. 2. Co-localization of DJ-1 with mitochondrial complex I.}

A. SH-SY5Y cells were stained with Mito Tracker Red and then with an anti-DJ-1 antibody. Nuclei were stained with DAPI. Cells were then reacted with FITC- or rhodamine-conjugated secondary antibody and images were visualized under a fluorescence microscope as described 
in Materials and methods.

B. NIH3T3 cells were heated at $95^{\circ}$ for 15 min and stained with anti-DJ-1 and anti-NDFFS3 antibodies. Cells were then reacted with FITC- or rhodamine-conjugated secondary antibody and images were visualized under a fluorescence microscope as described in Materials and methods.

Fig. 3. Reduction of complex I activity in DJ-1-knockdown cells.

A. Mitochondria were prepared from NIH3T3 and D2 cells and their complex I activities were measured.

B. Proteins in mitochondria used for measuring complex I activity in A were analyzed by Western blotting with anti-DJ-1, anti-NDUFA9, anti-NDUFS3, anti-ND6, anti-ATP5A1 and anti-actin antibodies as described in Materials and methods.

C. Left panel: Tet-DJ-1-knockdown cells and vector cells were cultured in the presence or absence of $1 \mu \mathrm{g} / \mathrm{ml}$ doxycycline for $72 \mathrm{hrs}$. Proteins in cells were analyzed by Western blotting with anti-DJ-1 and anti-actin antibodies. Right panel: Intensities of bands corresponding to DJ-1 in the left panel were quantified, and intensity of DJ-1 in cells without addition of doxycycline was set at 1 and relative intensity of each band is represented.

D. Tet-DJ-1-knockdown cells and vector cells were cultured in the presence or absence of 1 $\mu \mathrm{g} / \mathrm{ml}$ doxycycline for $72 \mathrm{hrs}$. Then mitochondria were prepared from cells and their complex I activities were measured. Significance: $* P<0.05, * * P<0.01$. N.S. indicates "non-specific". E. Proteins in mitochondria used for measuring complex I activity were analyzed by Western blotting with anti-DJ-1, anti-ATG5A1 and anti-actin antibodies. 
Fig. 4. Restoration of complex 1 activity in D2 cells expressing human DJ-1.

A. 293T cells were transfected with expression vectors for Flag-NDUFA4 and HA-tagged wild-type or L166P DJ-1. At 48 hrs after transfection, proteins were extracted from transfected cells and immunoprecipitated with an anti-Flag antibody or IgG, and precipitates were analyzed by Western blotting with anti-HA and anti-Flag antibodies.

B. Mitochondria were prepared from NIH3T3, D2 cells and D2 cells expressing wild-type or L166P human DJ-1 and their complex I activities were measured. Significance: $* P<0.05$. 
A

DJ-1

Mitochondria
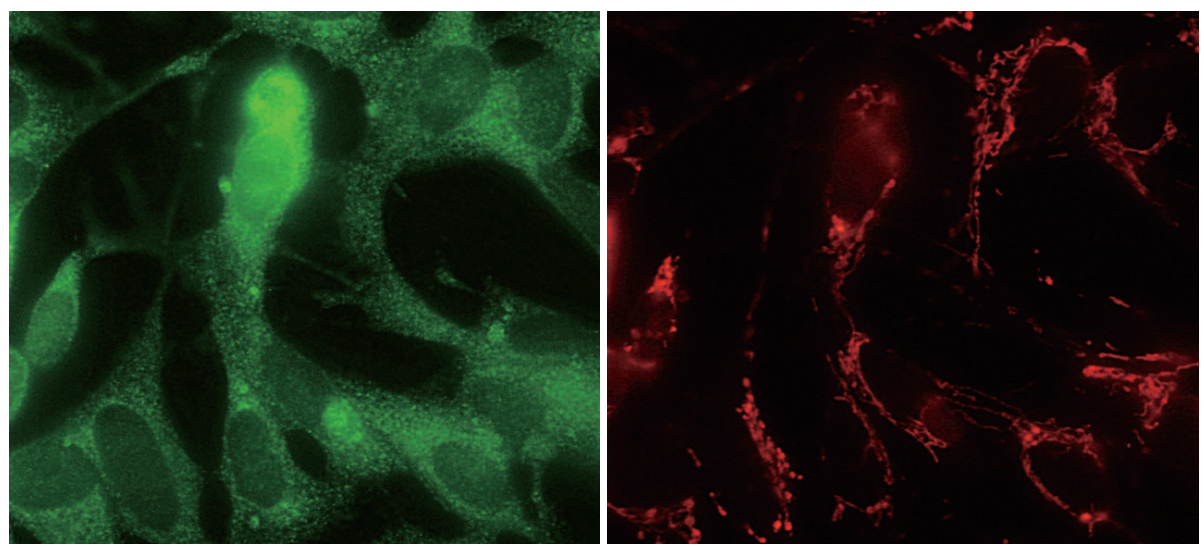

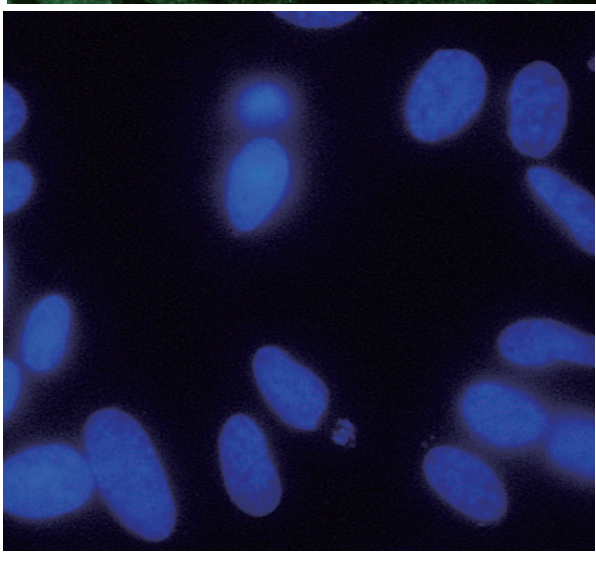

DAPI

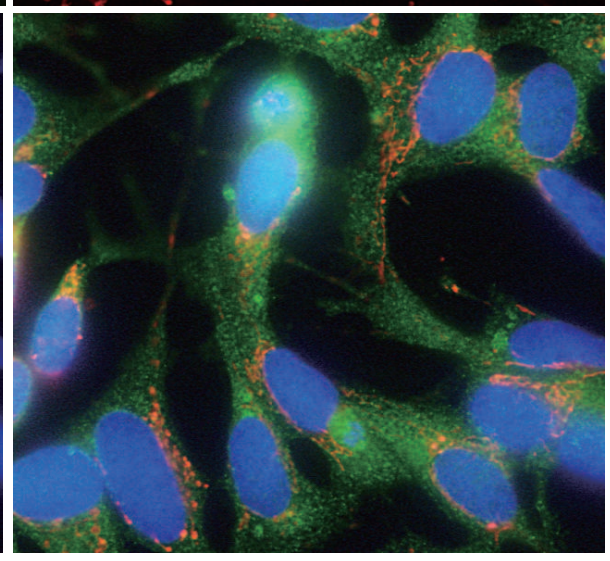

Merge

B

DJ-1

Complex I / NDUFS3
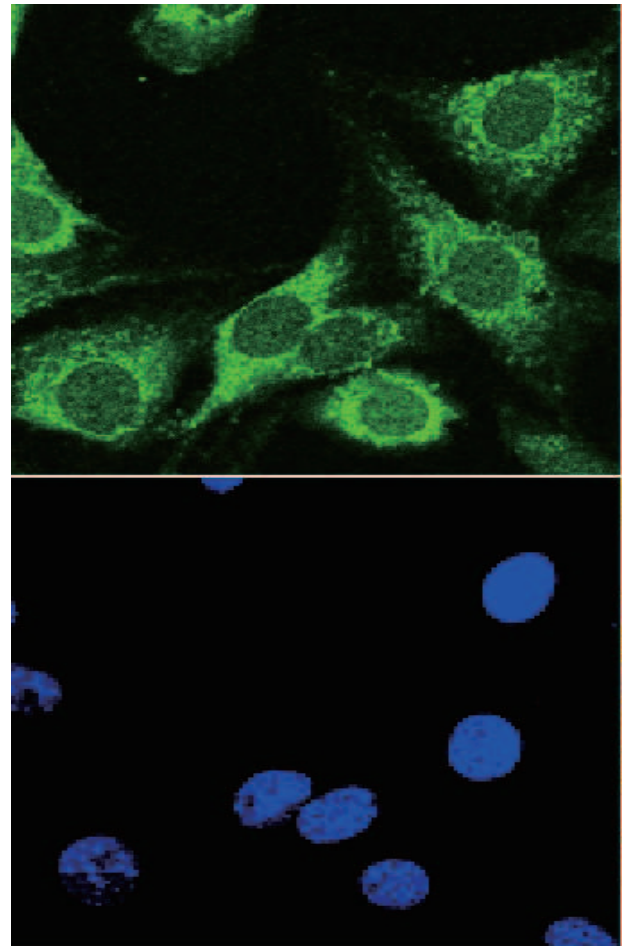

DAPI

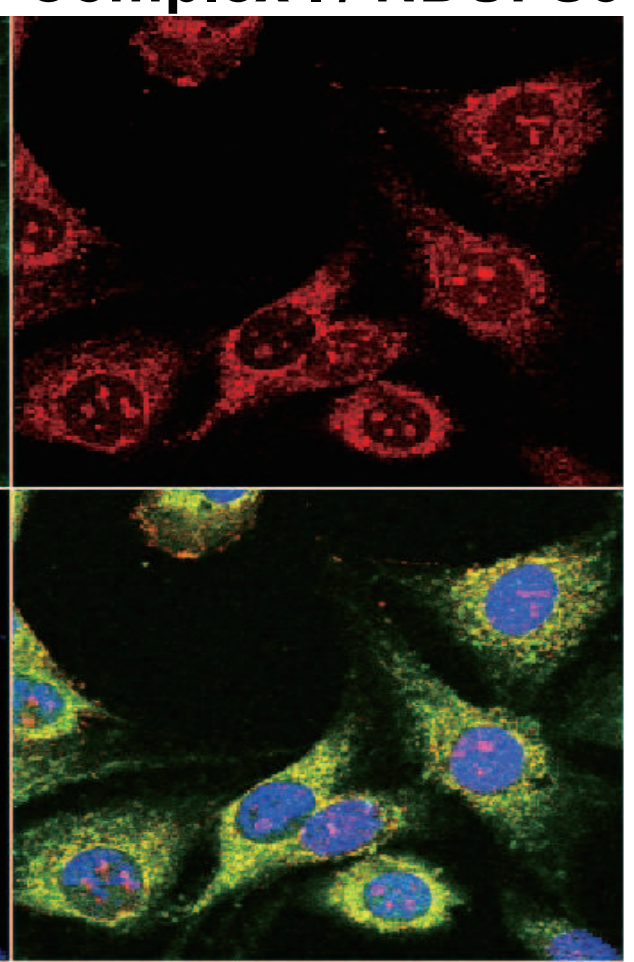

Merge

Fig. 2 

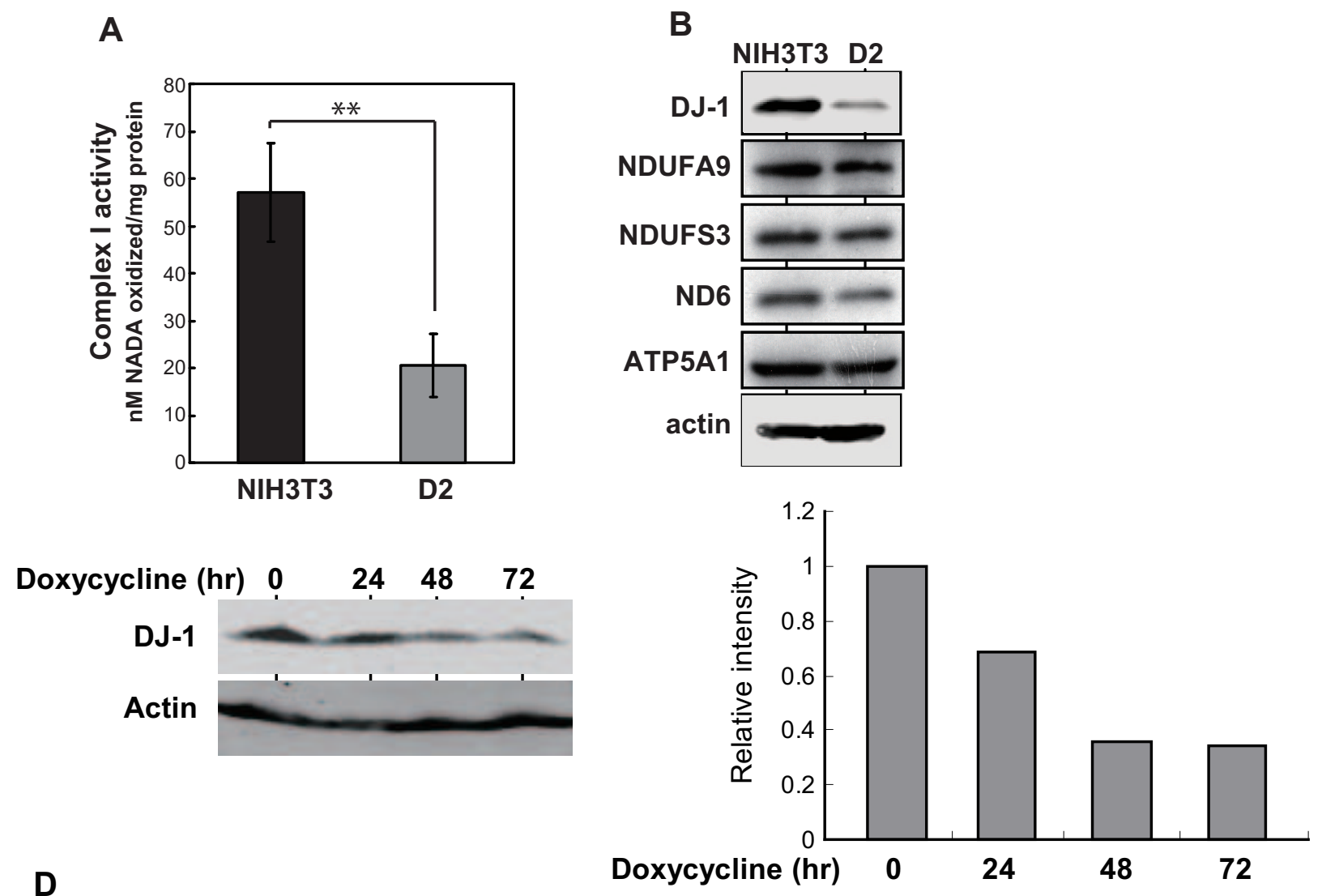

D

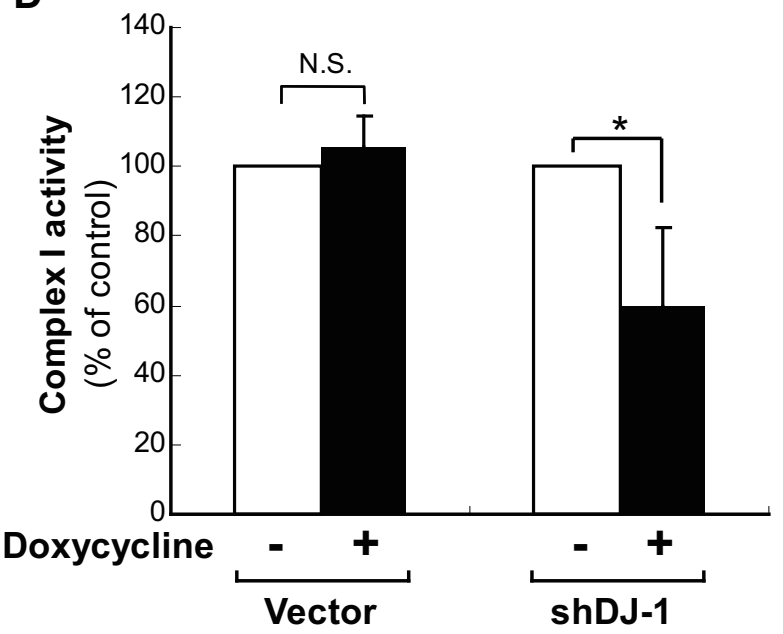

E

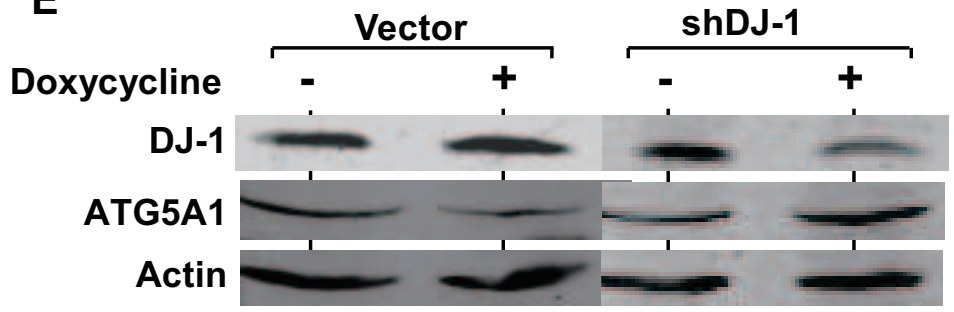

Fig. 3 
